\title{
Effectiveness of text messaging interventions on prevention, detection, treatment, and knowledge outcomes for sexually transmitted infections (STIS)/HIV: a systematic review and meta-analysis
}

Darlene Taylor ${ }^{1 *}$, Carole Lunny ${ }^{2}$, Petra Lolić ${ }^{3}$, Orion Warje ${ }^{4}$, Jasmina Geldman ${ }^{1}$, Tom Wong ${ }^{5}$, Mark Gilbert ${ }^{3}$, Richard Lester ${ }^{6}$ and Gina Ogilvie ${ }^{7}$

\begin{abstract}
Background: Rates of STIs continue to rise worldwide, and novel evidence-based interventions such as text messaging aimed at improving client services are needed. We conducted a meta-analysis to evaluate text messaging to support STI/ HIV prevention and treatment interventions.

Methods: We included articles that reported findings from randomized controlled trials (RTCs) involving adults and youth who were at risk of acquiring (or who currently had) a STI and/or HIV, a text message and comparator intervention, and reported provided outcome data on adherence to STI/HIV treatments. Articles were excluded if they were not published in English. We only included studies that have full-text publications so certainty and risk of bias assessments could be performed. Eight databases were searched to retrieve articles published between 1996 and March 2017. The Cochrane risk of bias tool was used and certainty of the evidence was assessed using GRADE. Effect estimates were pooled using a random effects model.

Results: A total of 35 RCTs were found, 6 of which were considered at low risk of bias. Eight studies found an increased association using text messaging in appointments attended compared to standard care (OR 1.64, 95\% Cl 1.28 to 2.10). Participants receiving text messages had an increase in HIV testing compared to standard care $(n=6 ; \mathrm{OR} 1.73,95 \% \mathrm{Cl} 1.39$ to 2.15). Ten text messaging RCTs measuring adherence using micro-electro-mechanical systems (MEMS) pill counts has a non-significant association (OR 1.17, 95\% Cl 0.95-1.45) while five studies measuring adherence by self-report was found to be significant (OR 1.64, 95\% Cl 1.28-2.11).
\end{abstract}

Conclusions: The effectiveness of text message interventions is equivocal. While text messaging has the potential to enhance the delivery of STI/HIV interventions, program planners are encouraged to evaluate any SMS intervention to ensure it is achieving the desired result.

Systematic review registration: PROSPERO CRD42013006503

Keywords: Text messaging, Sexually transmitted diseases, Delivery of health care, Evidence-based medicine, Meta-analysis

\footnotetext{
* Correspondence: darlene.taylor@ubc.ca

${ }^{1}$ University of British Columbia, 1147 Research Road, Rm ARTS 154, Kelowna,

BC V1V 1V7, Canada

Full list of author information is available at the end of the article
}

(c) The Author(s). 2019 Open Access This article is distributed under the terms of the Creative Commons Attribution 4.0 International License (http://creativecommons.org/licenses/by/4.0/), which permits unrestricted use, distribution, and reproduction in any medium, provided you give appropriate credit to the original author(s) and the source, provide a link to the Creative Commons license, and indicate if changes were made. The Creative Commons Public Domain Dedication waiver (http://creativecommons.org/publicdomain/zero/1.0/) applies to the data made available in this article, unless otherwise stated. 


\section{Background}

Increasing rates of sexually transmitted infections (STIs) remain a major public health challenge worldwide, posing a challenge to its control and appropriate management. According to the World Health Organization (WHO), "more than 1 million STIs are acquired every day worldwide" with a yearly incidence rate of 357 million per year [1]. In many western jurisdictions including Canada, Australia, and the USA, reported rates of genital chlamydia and gonorrhea are increasing. In Canada, for example, rates of genital chlamydia have steadily increased from 133/100,000 population in 1999 to 304/ 100,000 in 2015 [2]. Similarly, the rate of gonorrhea infections increased from 20/100,000 reported cases in 1999 to 50/100,000 reported cases in 2015 [2]. The latest data from Australia shows that gonorrhea has increased by $63 \%$ over the past 5 years (between 2012 and 2016; 62 to 101 per 100,000) [3]. These increased rates heighten concerns of sequelae such as pelvic inflammatory disease (PID), infertility, and increased vulnerability to acquiring HIV. Furthermore, rates of reported cases of infectious syphilis, continue to rapidly increase among men who have sex with men (MSM) in Canada, the USA, and Western Europe [4-6].

Surveillance, prevention, and control of chlamydia, gonorrhea, syphilis, herpes, human papillomavirus (HPV), and human immunodeficiency virus (HIV) infections consume a substantial direct cost [7]. In contrast, text messaging interventions may be cost-effective and can potentially reach a large number of people across large jurisdictions $[8,9]$. Evidence-based prevention and control interventions are key to controlling these communicable diseases [10].

Cell phones are widely used globally with an estimated $62.9 \%$ of the population worldwide owning a mobile phone. This estimate is expected to exceed five billion by 2019 [11]. As such, the popularity of this technology, including the capability to transmit text messages (also referred to as short message service [SMS]), may provide an opportunity to reach populations. SMS has become the most common mode of communication among almost five billion mobile phone users worldwide [12] and provides an easy way for health staff to administer an intervention. Text messages have been used to remind patients about clinic appointments, to notify patients that it is time for STI re-testing, and to facilitate patient communication with their health professionals related to their sexual health. Text messaging can be delivered manually or through automated systems [13] and can act as an intervention via two-way communication, which can allow for interactive support by health care providers, or via one-way program-initiated communication to deliver key health messages to the user [14-16].
A large body of literature has emerged demonstrating the effectiveness of SMS on STI/HIV control [17-20]. However, individual study results are markedly disparate. This points to the need for a comprehensive systematic review and meta-analysis. Several systematic reviews have been published examining SMS interventions in relation to broad questions such as attendance in clinics [21], sexual health in adolescents [22-24], reminder interventions to retest [25], and countless others. Three previous meta-analyses [26-28] and one network metaanalysis [29] have been conducted that pooled the results of randomized controlled trials (RCTs) on the effectiveness of text messaging interventions on HIV adherence and viral load. These programs take on varied purposes for engaging individuals through the prevent ion-to-treatment spectrum; however, the evidence of the effectiveness of text messaging interventions must be clear before such programs could be transitioned to policy and recommended as a tool for STI prevention and control globally.

We are improving previous research by including and reviewing more current studies, including only RCTs, assessing the risk of bias of the included RCTs, and grading the overall certainty of evidence [30]. We conducted a systematic review and meta-analysis of RCTs aimed at evaluating the impact of SMS on STI/HIV prevention, detection, and control.

\section{Methods}

This systematic review was registered with PROSPERO (CRD42013006503), the protocol has been published [31], and a PRISMA statement checklist has been completed (Additional file 1).

\section{Search strategy}

This systematic review and meta-analysis was performed according to the Preferred Reporting Items for Systematic review and Meta-Analysis (PRISMA) guidelines [32]. The search was conducted in two phases in order to provide an up-to-date review. The initial search (phase 1) was conducted in the Cochrane Database of Systematic Reviews, MEDLINE, ACP Journal Club, Database of Abstracts of Reviews, EMBASE, EBM Reviews, and included articles published from 1996 to August 31, 2013. We chose to limit the search starting at 1996 as it was thought that this was when SMS first became widely used. In addition, the table of contents for the following journals was reviewed: Sexually Transmitted Diseases, Sexually Transmitted Infections, and AIDS Patient Care and STDs. Grey literature was searched using Google and Grey Literature Report (www.greylit.org) to identify studies that may have been missed in the abovementioned searches. Medical subject headings $(\mathrm{MeSH})$ and keywords included mobile health, mHealth, cell phone, 
mobile phone short message services, text messaging, texting, SMS, MMS, communication technologies, patient monitoring devices, wireless technologies, STI testing, sexually transmitted diseases, sexually transmitted infections, HIV, chlamydia, gonorrhea, herpes, Trichomonas vaginalis, and syphilis. The search strategies are included in Additional file 2. The second search (phase 2) was conducted in MEDLINE and mhealthevidence.org (Additional file 3) using the same search terms. Limiting our search to MEDLINE and mhealthevidence.org was done in this phase due to a lack of resources. This included articles published between September 1, 2013, and March 30, 2017. We also examined the reference lists of any relevant systematic reviews retrieved.

\section{Eligibility criteria}

RCTs examining the effect of SMS on STI/HIV prevention and control outcomes among adults and youth published in English between January 1, 1996, and March 30, 2017, were included. For phase 2, we only included studies that were identified in MEDLINE and mhealthevidence.org between 2013 and 2017. For the purposes of this review, SMS was defined as a text message that is delivered to a mobile phone either manually or by an automated system. We included only RCTs published in English as we did not have the financial and human resources to translate non-English studies. A detailed description of the inclusion/exclusion criteria using a PICO (Population, Intervention, Comparison, Outcome) format can be found in Additional file 4 .

\section{Selection of studies}

Titles and abstracts were independently examined for eligibility by two reviewers (PL, OW). Disagreements were resolved by a third reviewer (DT). Studies that were deemed eligible based on title and abstract were then reviewed by two independent researchers to confirm eligibility. Forward searching was done using the included references. Our updated search involved screening by one reviewer (CL) with a second reviewer checking the full text of included studies (DT). No test of agreement between reviewers was conducted for either of the search phases. All discrepancies were discussed until consensus was achieved.

\section{Data extraction}

Data were extracted independently by two reviewers (PL, OW) using a standardized pre-tested data extraction form. Data extracted included (1) purpose of intervention, (2) duration of intervention, (3) frequency of text messages, (4) setting, (5) intervention, (6) comparison, (7) one way or two-way SMS, (8) type of participants, (9) follow-up period, (10) outcome results, and (11) reported effect measure (risk ratio, odds ratio, etc.) from the study. The studies included in our updated search were extracted by one reviewer (CL) and 100\% checked by a second reviewer (DT). No test of agreement between reviewers was conducted for either of the search phases. All discrepancies were discussed until consensus was achieved.

\section{Risk of bias assessment}

Risk of bias was assessed using Cochrane's risk of bias (ROB) tool [33]. In Cochrane's "other" ROB domain, we included "incomplete outcome data (intention to treat [ITT] analysis)." This was defined as randomized participants having been analyzed according to their allocated treatment, irrespective of whether they were eligible, received the allocated treatment, received another treatment, or received no treatment. Study certainty was rated independently by PL and OW, and any discrepancies were discussed until consensus was reached. The studies included from our updated search involved assessment by one reviewer (CL) and checking by a second reviewer (DT). RCTs were considered at low risk of bias if they were rated at low risk for the following domains: random sequence generation, allocation concealment, incomplete outcome data (attrition bias and intention-to-treat [ITT] analysis), and selective reporting bias.

\section{Unit of analysis issues}

As recommended in the Cochrane Handbook [33], we combined multiple intervention arms to overcome unit-of-analysis error and to create a single pair-wise comparison.

\section{Certainty of the evidence assessment}

We graded the overall certainty of evidence using the Grading of Recommendations Assessment, Development and Evaluation (GRADE) approach [30]. GRADE assessments were conducted by a single reviewer. GRADEpro software [34] was used to create Summary of Findings tables for the following primary outcomes: appointment adherence, adherence to ART by MEMS (medication event monitoring system) pill count, and adherence to ART by self-report. The certainty of the evidence was categorized as high certainty, moderate certainty, low certainty, or very low certainty based on a judgment of the confidence in the effect estimate, whether the true effect is likely to be substantially different from the estimate of effect. The certainty of the evidence is downgraded from high, moderate, low to very low for the following reasons: risk of bias, inconsistency (unexplained heterogeneity, inconsistency of results), indirectness of evidence (indirect population, intervention, control, outcomes), imprecision of results (wide confidence intervals), and risk of publication bias. 


\section{Analysis}

Clinical heterogeneity was assessed within each pairwise comparison by comparing characteristics [33]. In order to minimize heterogeneity, outcomes were categorized as follows: (1) SMS used for prevention of STIs/HIV, (2) SMS used to impact adherence to HIV medication adherence, and (3) SMS used to impact HIV treatment outcomes. Sub-analyses were conducted based on these different categories. As the characteristics of the 35 included studies were variable, we chose to use the Mantel-Haenszel random effects model. Statistical heterogeneity was examined using the $I^{2}$ statistic and interpreted as an $I^{2}$ estimate of $0 \%=$ no heterogeneity, $25 \%=$ low, $50 \%=$ moderate, and $75 \%=$ high heterogeneity of effect sizes [33]. We summarized dichotomous outcomes using odds ratios (OR), with 95\% confidence intervals (CI). We used RevMan 5.3 to conduct the statistical analysis and graphs.

\section{Publication bias}

If more than ten studies reported the same outcome, publication bias was explored with the Egger test and a funnel plot using STATA 13 software with the metan, metafunnel, metabias, and metatrim packages [35-39]. A $p$ value less than 0.05 was considered statistically significant. Stata's trim and fill method was used to estimate the number of missing null studies from the meta-analysis. Stata's metatrim command performs the Duval and Tweedie nonparametric "trim and fill" method of accounting for publication bias in meta-analysis. The method, a rank-based data augmentation technique, formalizes the use of funnel plots, estimates the number and outcomes of missing studies, and adjusts the meta-analysis to incorporate the theoretical missing studies.

Sensitivity analyses were conducted with and without statistical outliers to assess their effect on the overall findings. In addition, a sensitivity analysis was conducted to assess the impact of RCTs judged to have low methodological certainty on the summary effect. If three or more studies exist in each group, we subgrouped adherence as "self-reported" and "objectively-measured" adherence (MEMS pill count).

\section{Results}

A total of 14,850 articles were initially identified through database and manual searches conducted between 1996 and March 2017 (both phases) (Fig. 1). Four thousand six hundred eighty duplicates were removed, and 9949 were not included as they were not relevant to our topic. Of the remaining 221 full text articles, 186 were excluded because they were case studies $(n=2)$, duplicate records of the same study ( $n$
$=9$ ), included no data and authors were unreachable $(n=15)$, had no relevant outcomes $(n=40)$, did not involve texting $(n=22)$, both arms received text messages $(n=3)$, was not a full article $(n=1)$, and not a RCT $(n=94)$. The remaining 35 RCTs [40-73] were included. Table 1 displays the characteristics of the 35 included studies. A description of the content of the SMS messages can be found in Additional file 5 .

Of the 35 studies included in this systematic review, the majority investigated either the effectiveness of text messaging to support STI clinic appointment attendance (8 studies), or the effectiveness of text messaging to support HIV medication adherence (15 studies). These studies were conducted in 14 countries, the majority being in the USA (13 studies), Kenya (5 studies), and South Africa (3 studies) and were conducted in a variety of settings including clinics (19 studies), hospitals (6 studies), universities (4 studies), and others such as an emergency room ( 1 study) and a music festival (1 study). The age of participants ranged from 16 to 84 years and the length of follow-up ranged from 7 days to 24 months. Text messages were either sent daily, weekly, monthly, or as one-off reminders. The majority of studies (83\%) sent a one-way text with the remaining sending two-way texting.

\section{Risk of bias across studies}

The majority of studies (88.6\%) had low risk of random sequence generation using either minimisation, varying block size or computer-assisted randomization. Four studies had unclear risk of random sequence generation $[48,56,69,70]$. One study was at high risk of allocation concealment [18], 13 studies were at unclear risk [45, 47, $51,56,57,59,60,64,65,68-70,73]$, and the 21 remaining studies were at low risk.

In most studies, medical staff were masked to the knowledge of the allocated interventions; however, in the majority of cases, blinding of participants to intervention allocation was not done. Only two studies successfully blinded participants to the intervention [57, 67]. Participants in the Morris et al. [57] study were from a registry so they were blinded to their randomization group, and individuals in Rand et al.'s [67] control group received the same initial message as the intervention group, followed by a control message about a different general adolescent health topic each time reminders were sent to the intervention group; therefore, they were unlikely to ascertain their allocation.

High attrition bias was encountered in three studies $[40,56,73]$ and was unclear in three others $[45,46,70]$ as they had not recorded the number of participants lost to follow-up. Thirteen studies were at high risk of 


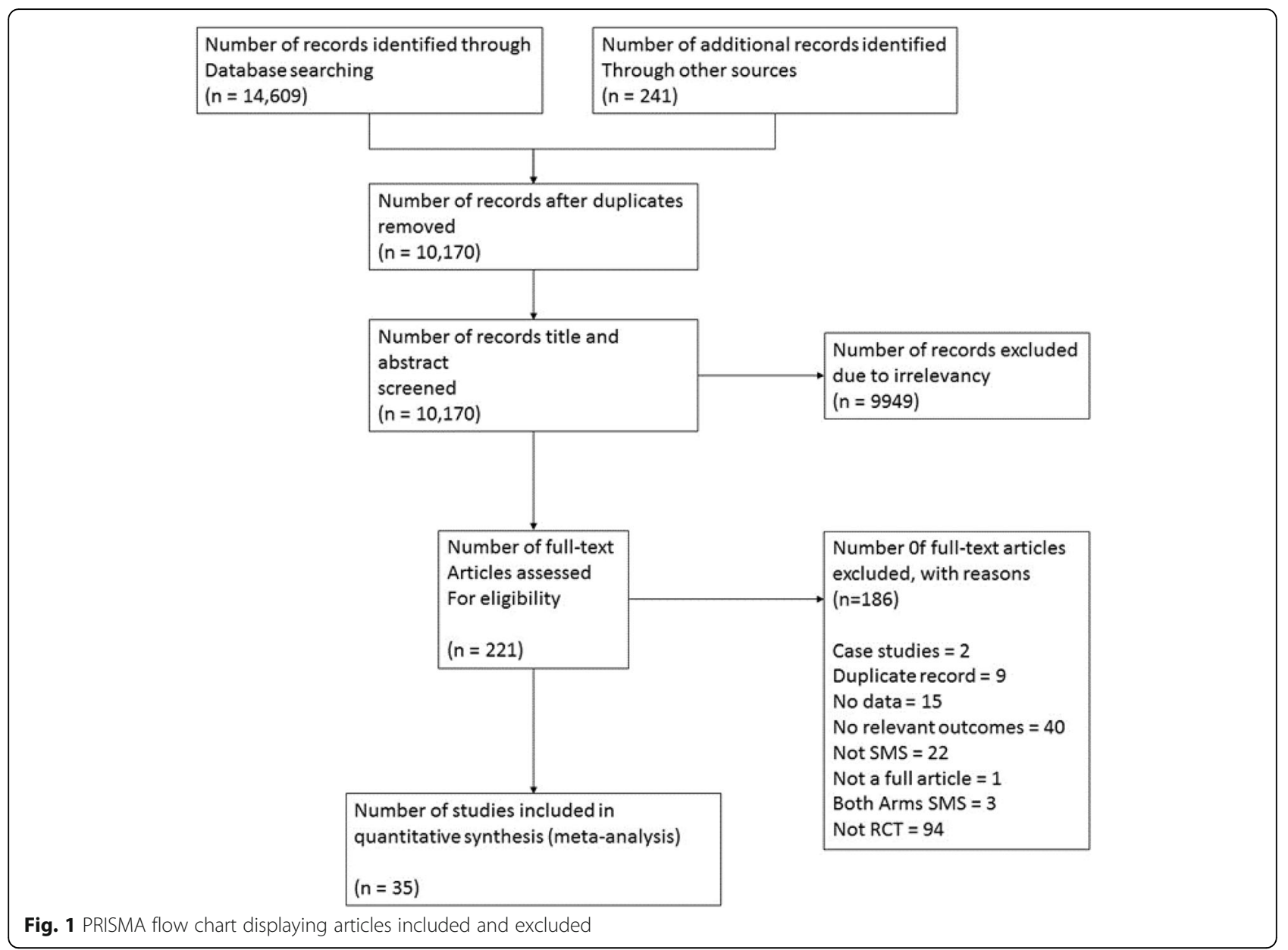

incomplete outcome bias (intention-to-treat [ITT] analysis) as they had not conducted the ITT analysis correctly or at all $[40,42,44,45,48,49,53,56,57,61,62$, $69,73]$. An intention-to-treat (ITT) analysis is recommended as the least biased way to estimate intervention effects in randomized trials [33].

Selective reporting bias was at high risk in three studies $[43,53,73]$ and at unclear risk in eight studies $[42,44,45,47,48,54,71,74]$ because of pre-protocol or registry information being unavailable. Six studies were considered at low risk of bias as they were rated at low risk for the following domains: random sequence generation, allocation concealment, incomplete outcome data (attrition bias and ITT analysis bias), and selective reporting bias [41, 50, 52, $63,66,68]$. Only three of these measured the same outcome (HIV adherence by pill count) and were therefore available for sensitivity analysis $[50,63,66]$. Risk of bias for each study is included in the forest plots provided (Figs. 2, 3, and 4). In addition, a summary graph of the risk of bias for all included studies and a detailed graph of the risk of bias of individual studies can be found in Additional file 6 .

\section{Outcomes}

Outcomes included in our analysis were divided into three categories: prevention interventions, HIV drug adherence interventions, and HIV treatment outcome interventions. A table organizing studies for subgroup analysis can be found in Additional file 7 .

\section{Prevention interventions (Fig. 2)}

Appointment adherence Eight studies [41, 43, 49, 58, $59,61,65,70]$ with a total of 5027 participants found an associated increase in appointment attendance among the SMS group compared to standard care (OR 1.64, 95\% CI 1.28 to 2.10 ). There was low heterogeneity among the pooled studies $\left(I^{2} 45 \%\right)$. Two studies that recruited youth (age $16-30$ years) had a pooled odds ratio of 2.03 with a wide confidence interval of 1.39 to 2.97 indicating imprecision $[58,70]$. In the case of Rutland et al.'s study [70], imprecision may have been a result of a small sample size.

Condom use Two studies [53, 73] with 1046 participants (aged between 16 and 29) examining the 


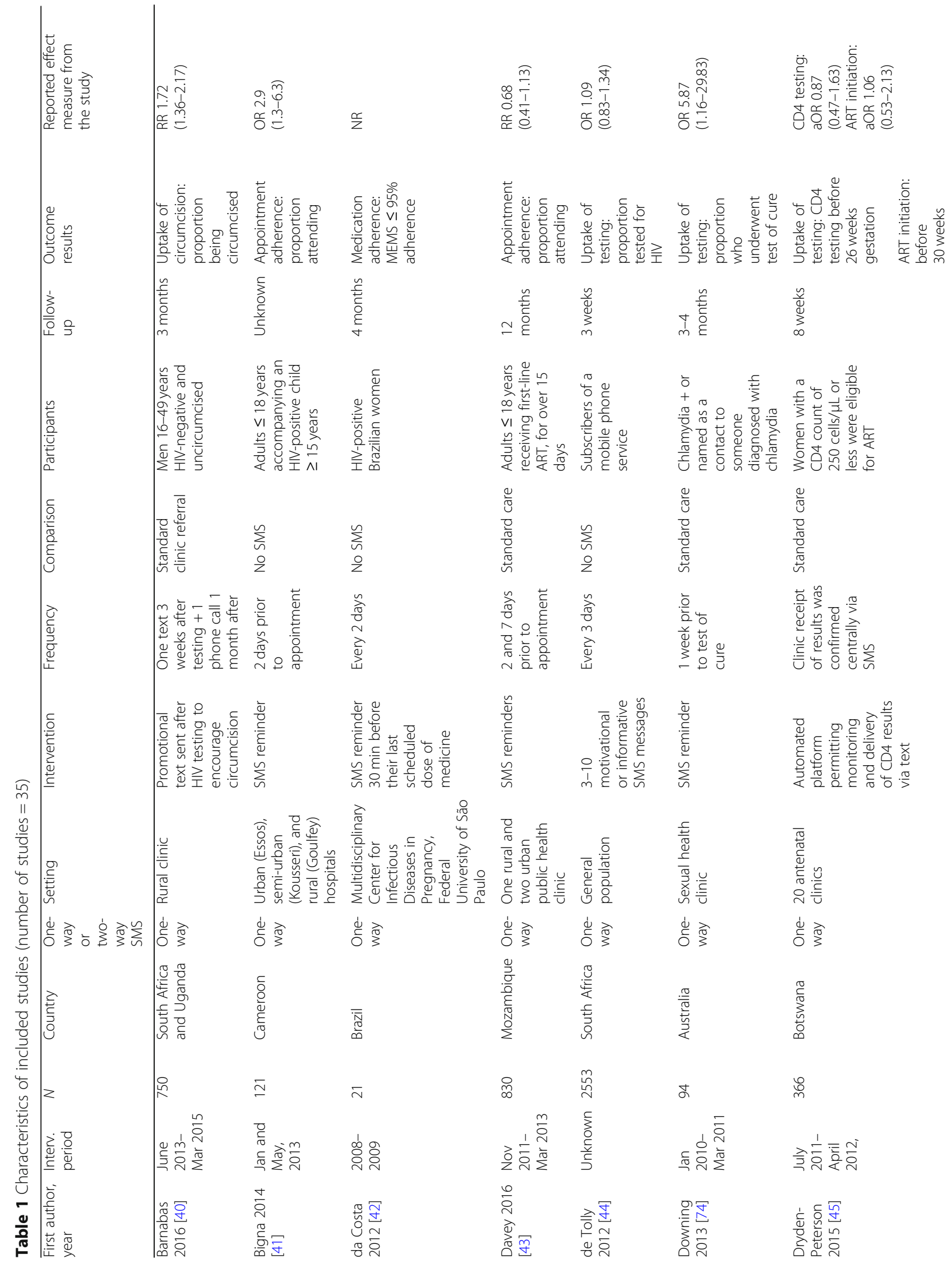




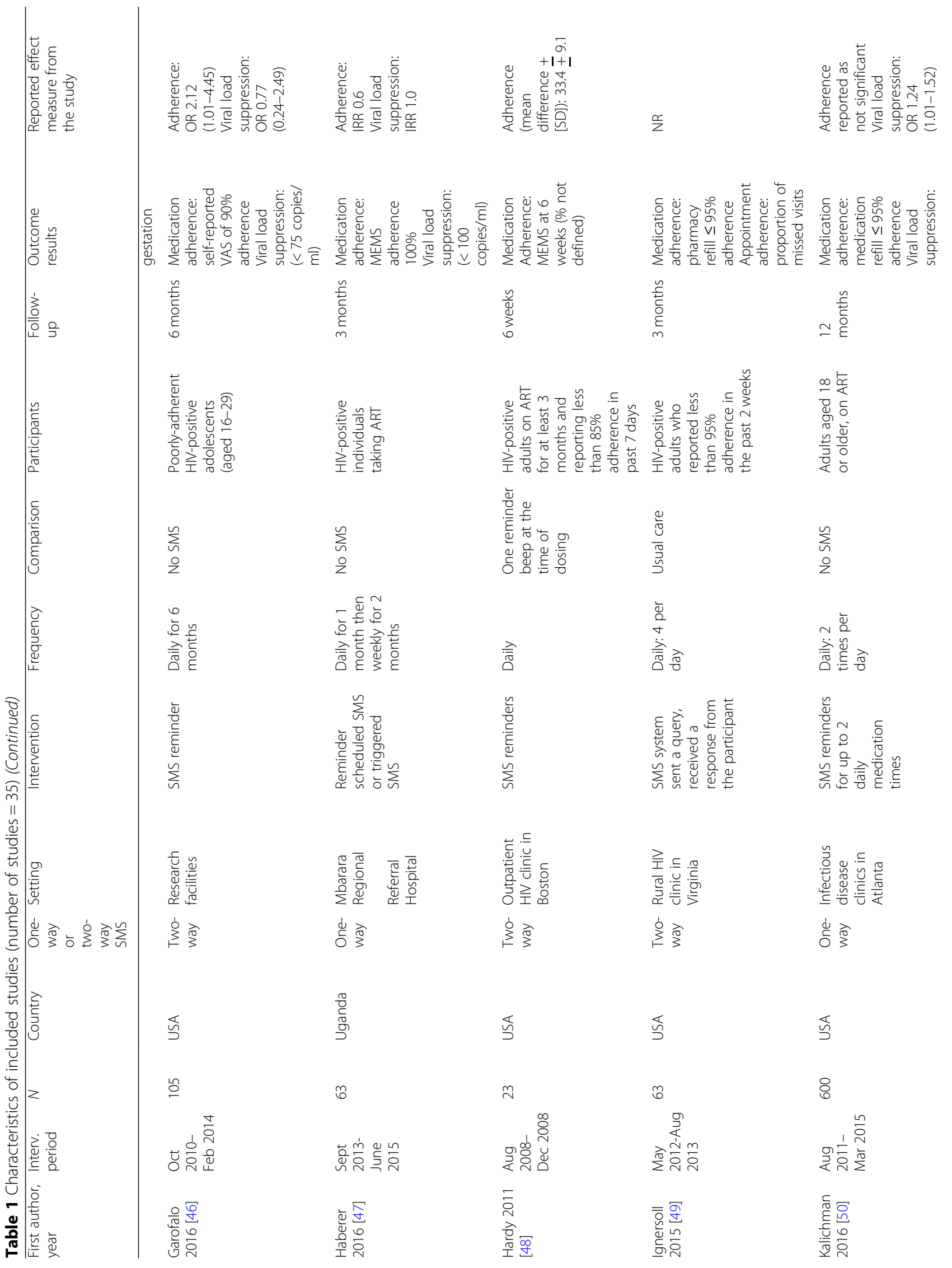




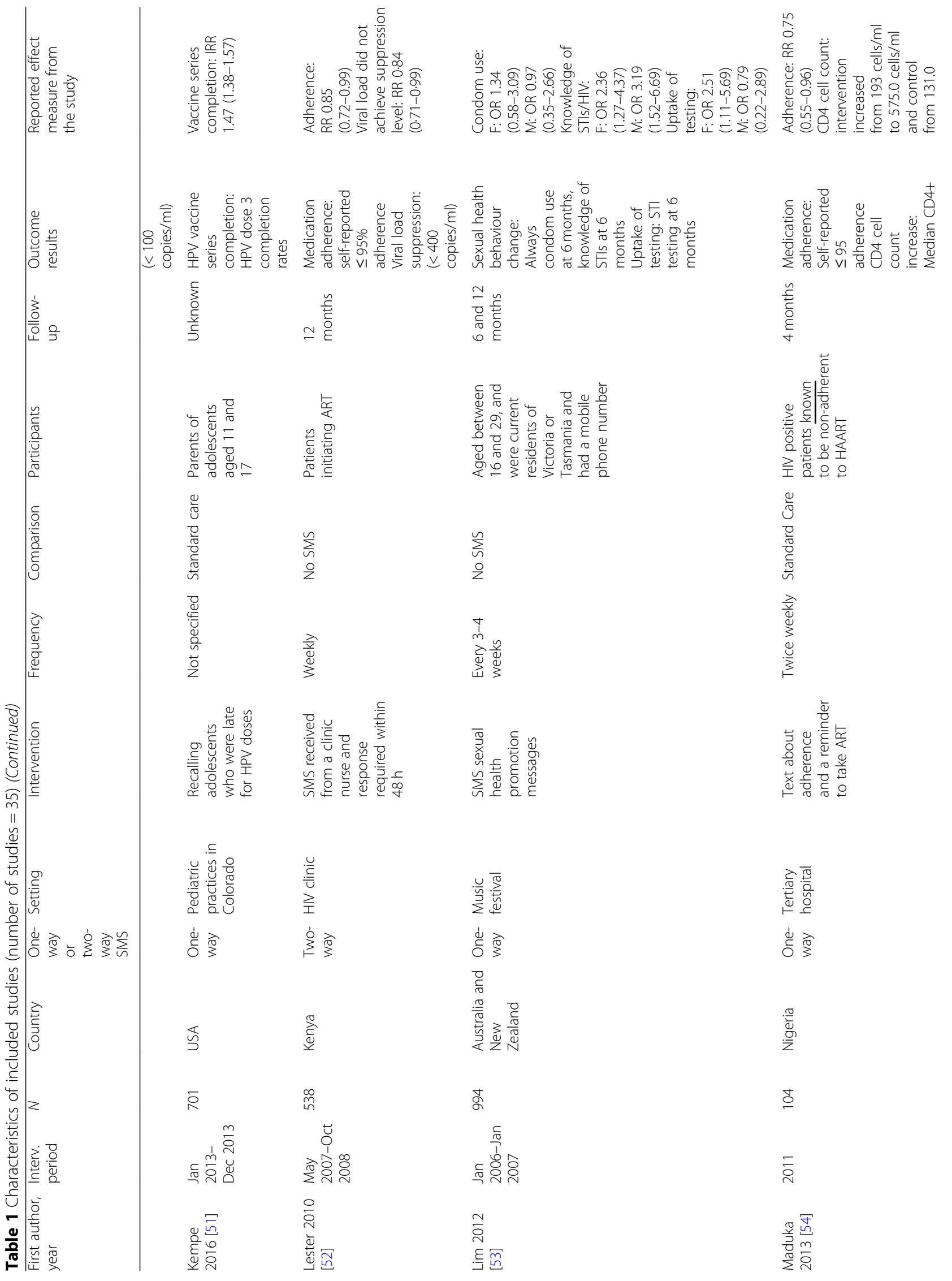




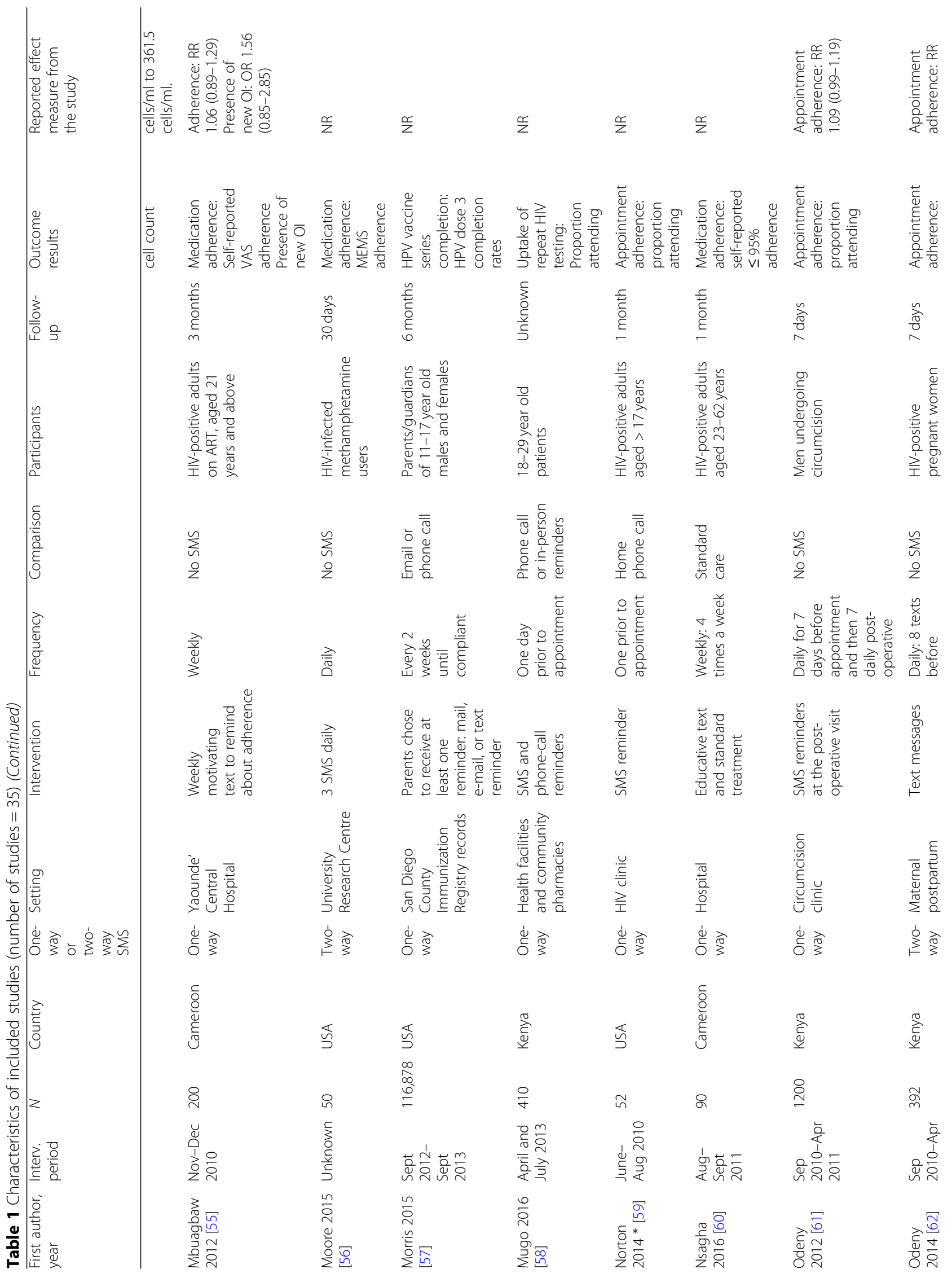




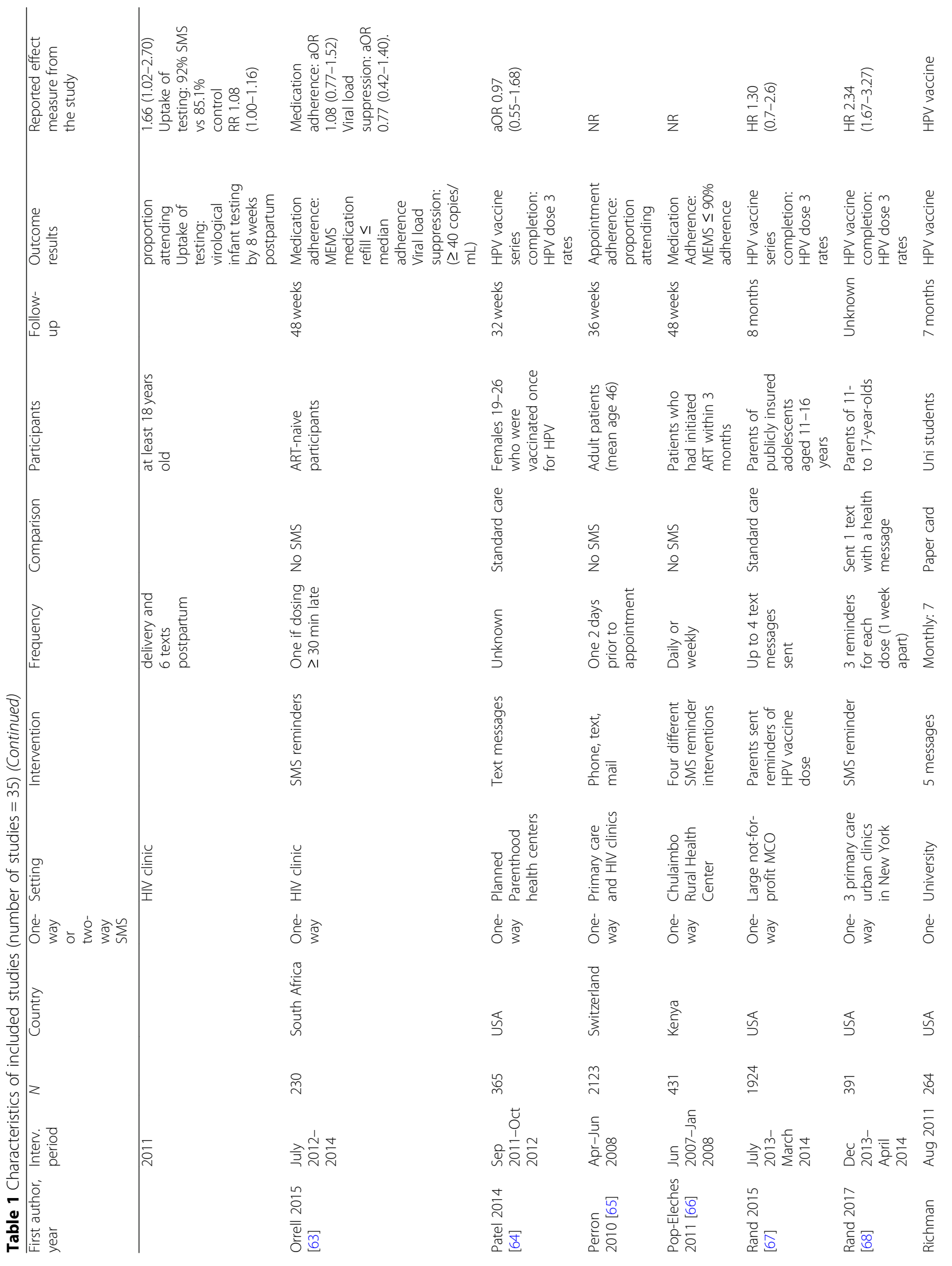




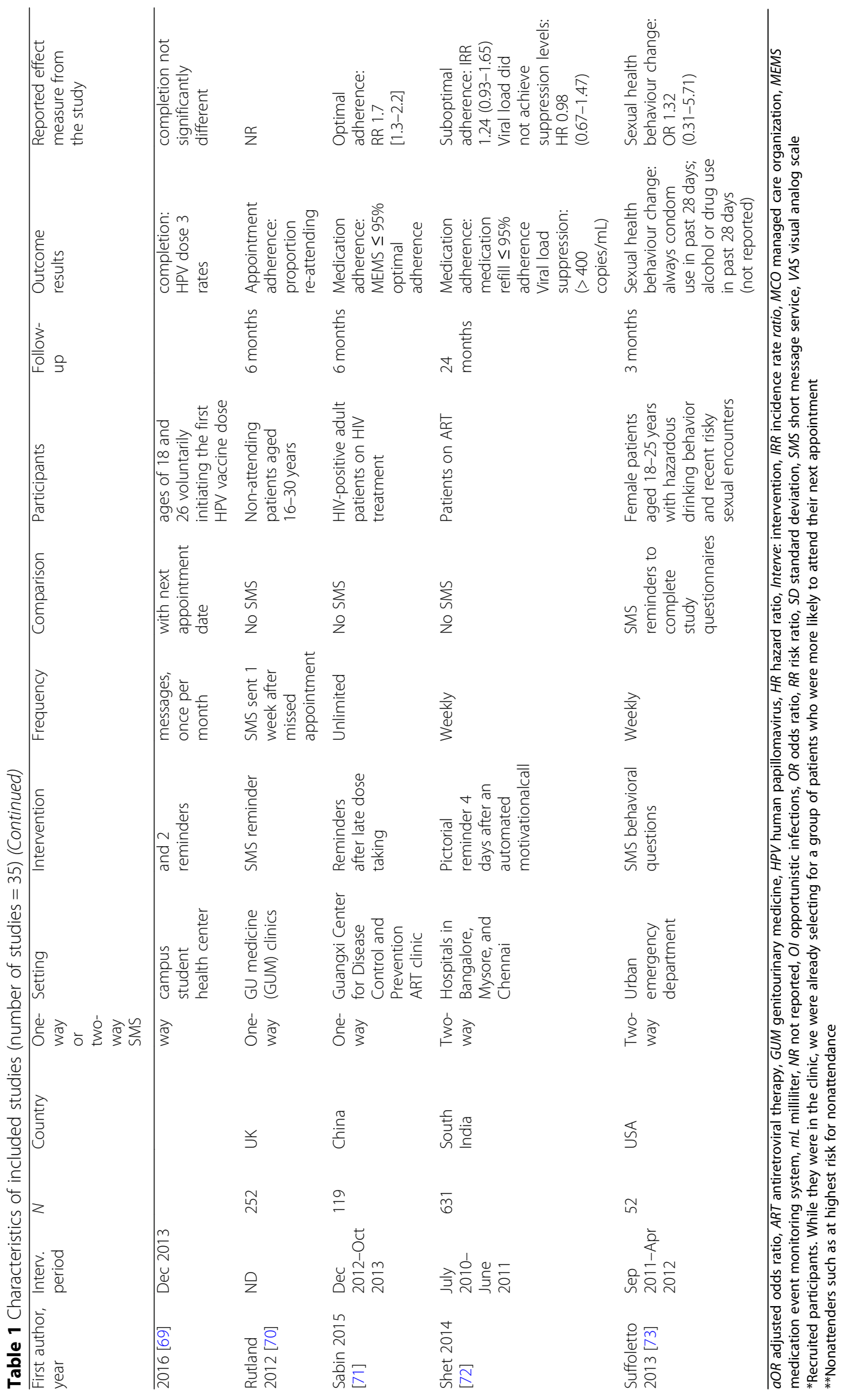




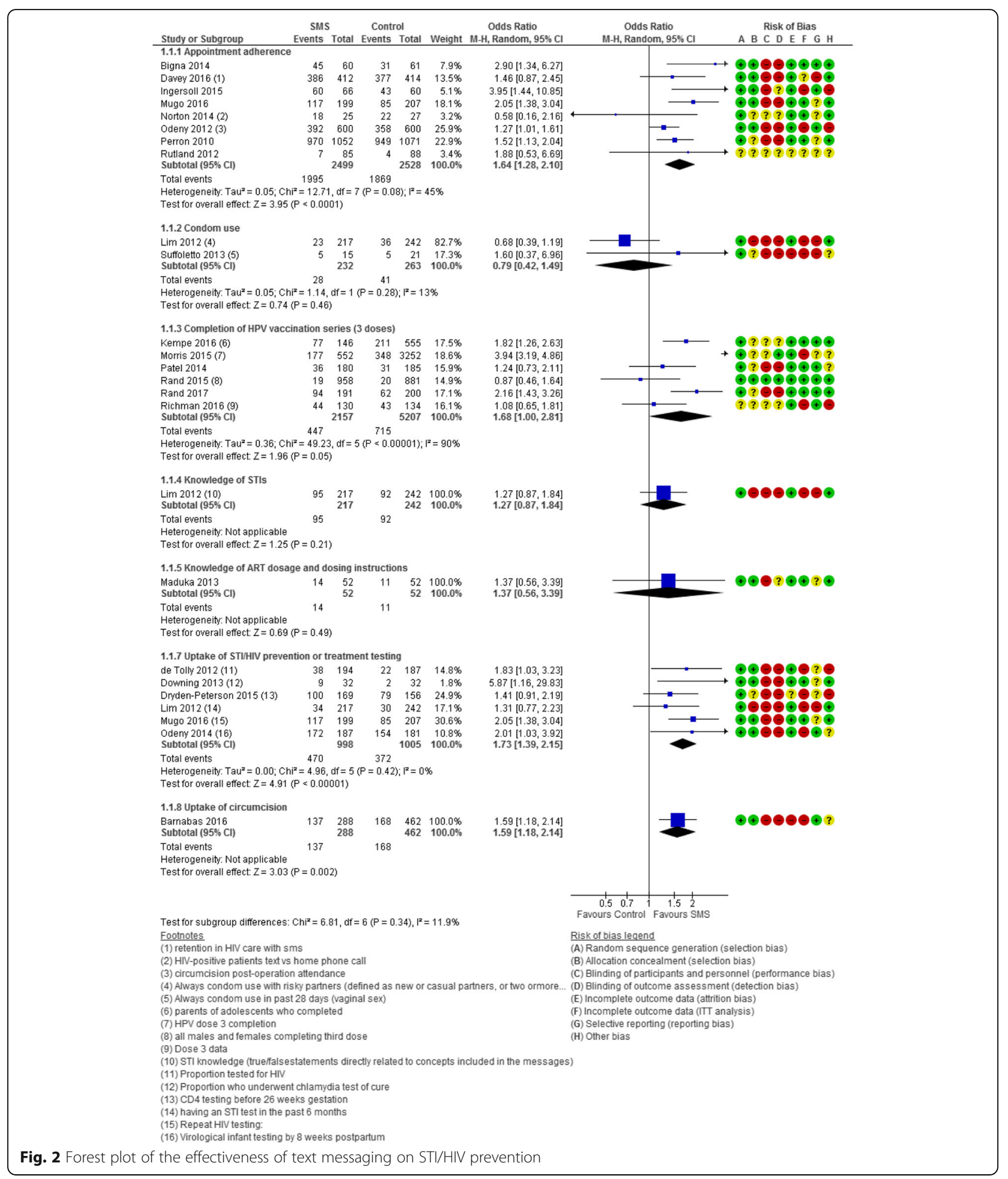

effectiveness of SMS on condom use reported no association between the intervention and control groups (OR $0.79,95 \%$ CI 0.42 to 1.49 ). This pooled effect was powered by Lim et al.'s study [53] ( $n=994$ enrolled; $54 \%$ attrition) that indicated condom use did not increase in the treatment group at 6 months follow-up compared to Suffoletto et al.'s study [73] ( $n=52$ females; $30 \%$ attrition) that reported a positive effect of the SMS intervention at 3 months follow-up. There was low statistical heterogeneity $\left(I^{2} 13 \%\right)$ among the two studies; however, 


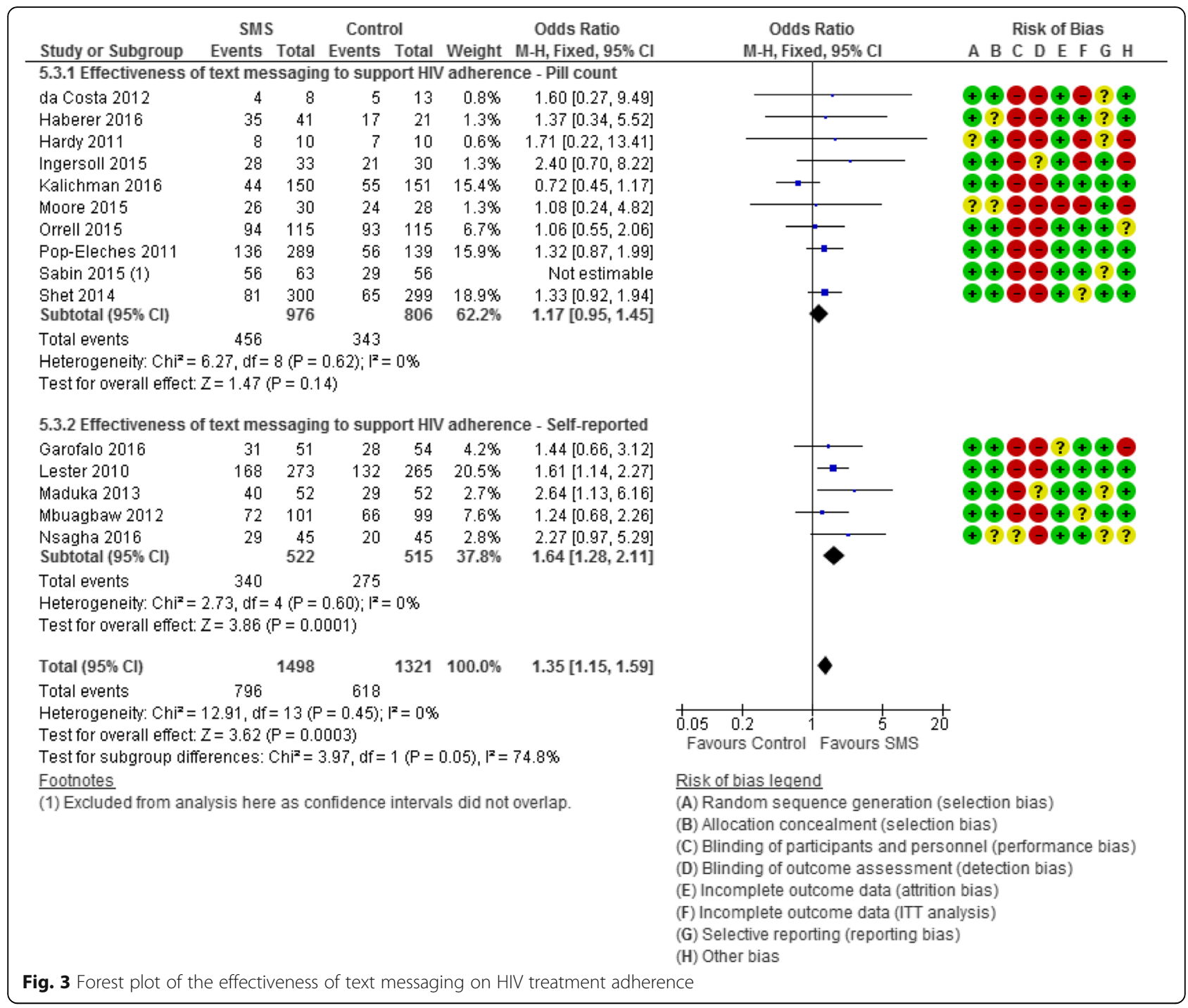

the two studies differed in the type of participants recruited and outcome definition. Lim et al. [53] recruited males and females at a music festival and defined condom use as always using condoms in the past 3 months with risky partners. In contrast, Suffoletto et al. [73] recruited females with hazardous drinking behavior and recent risky sexual encounters and defined condom use as always using condoms in the past month.

Uptake of STI/HIV testing Pooled results from six studies ( $n=4753$; $58 \%$ attrition) revealed a positive association between SMS and uptake of STI/HIV testing (OR $1.73,95 \%$ CI 1.39 to $2.15 ; I^{2} 0 \%$ ). Two studies [44, 58] evaluated the effectiveness of SMS on the uptake of HIV testing compared to standard care, one study [53] examined the effectiveness of SMS on the uptake of STI testing in the past 6 months compared to standard care, one study [74] examined those undergoing chlamydia test of cure, and two others examined CD4 testing before 26 weeks gestation [45] and viral load testing in infants by 8 weeks postpartum [62].

HPV vaccination series completion Six studies [51, 57, 64, 67-69] ( $n=10,301 ; 29 \%$ attrition) examined the effectiveness of text SMS compared to no reminders on completion of a three-dose HPV series. The pooled comparison found a non-significant association (OR 1.68, $95 \%$ CI 1.00 to 2.81 ) with high heterogeneity ( $\left.I^{2} 90 \%\right)$. One study by Morris et al. (OR 3.94, 95\% CI 3.19 to 4.86) was a clear outlier as its confidence intervals did not overlap with the other five studies, and when it was taken out of the pooled analysis, heterogeneity lowered to $I^{2} 55 \%$ and the confidence interval around effect estimate became more precise (OR 1.43, 95\% CI 1.04 to 1.97; Fig. 2). Two studies with populations aged between 18 and 26 were subgrouped and produced a pooled odds 


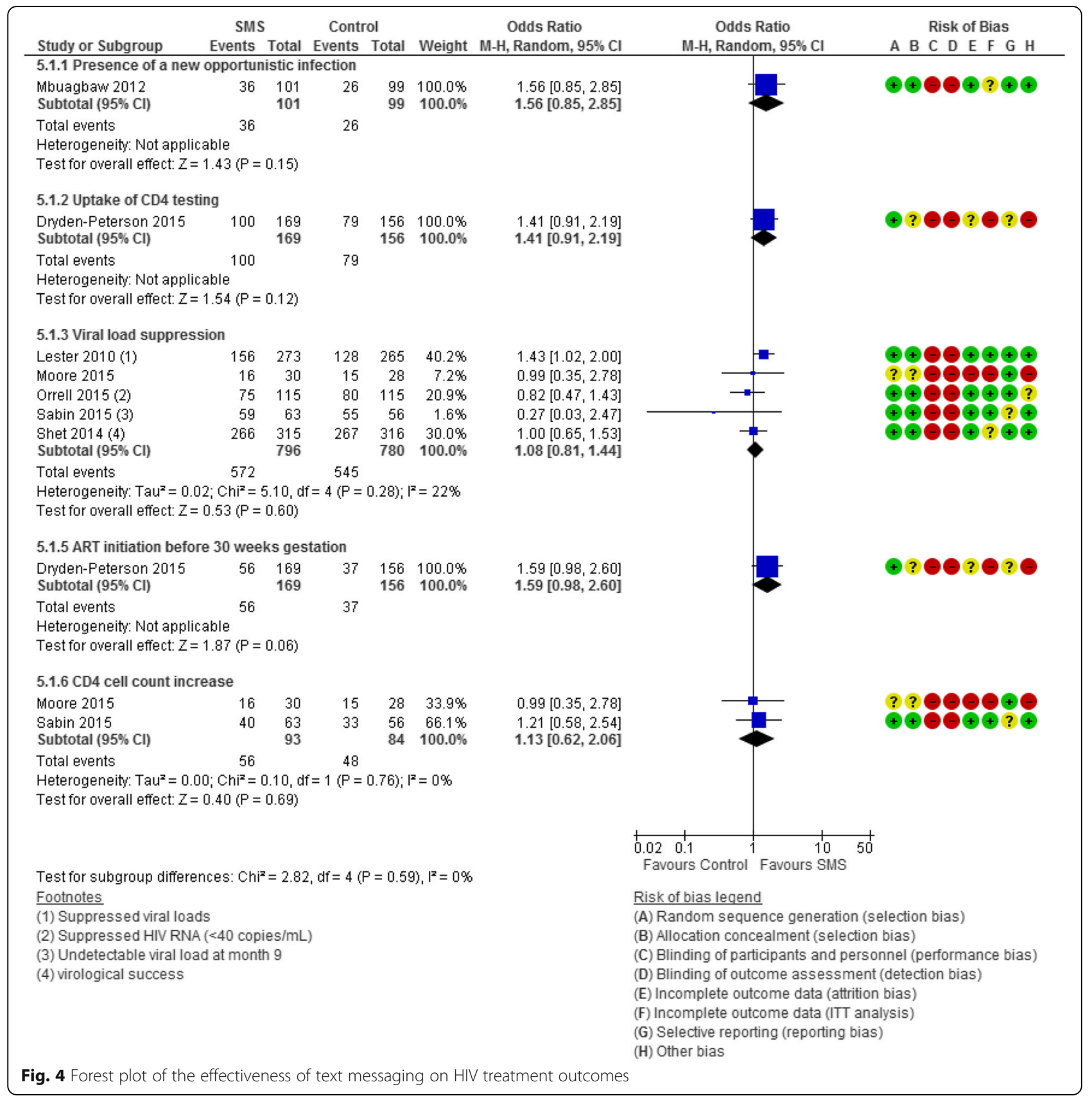

ratio of 1.16 (95\% CI 0.86 to 1.67 ) indicating no association between the intervention and control groups.

Knowledge of STIs, ART dosage, and instructions Two studies [53, 54] evaluated the effect of SMS on education surrounding STIs [53] and ART dosage and instructions [54] ( $n=1098 ; 49 \%$ attrition). Neither study showed a positive effect. Participants of the Maduka and Tobin-West study [54] did not have a statistically significant increase in knowledge of ART dosing and dosing instructions (OR 4.55, 95\% CI 0.92 to 22.55), nor with drug names (OR 1.37, 95\% CI
0.39 to 3.39$)$. However, the wide confidence interval (due to small sample size) in the knowledge of ART dosing and dosing instructions outcomes indicates substantial imprecision.

Circumcision One study [40] that examined the effectiveness of sending promotional texts to HIV-negative and uncircumcised men after HIV clinic testing to encourage circumcision compared to standard clinic referral found a positive association (OR 1.59, 95\% CI 1.18 to 2.14). However, another study of men who underwent circumcision [61] reported no association between men 
who resumed sex before the waiting period that was recommended by their doctor in the SMS group and the control group (RR 1.09, 95\% CI 0.99 to 1.19 ).

\section{Adherence to antiretroviral therapy (Fig. 3)}

Fifteen RCTs $(n=2819)$ examined the effectiveness of text messaging to support increased antiretroviral therapy (ART) adherence compared to standard care (no text). Ten of these studies measured adherence objectively using pill counts [42, 47-50, 56, 63, 66, 71, 72], and five used self-reported adherence $[46,52,54,55,60]$.

Overall, the pooled 15 text message interventions found a positive association with increased adherence compared to standard care (OR 1.43, 95\% CI 1.22 to $1.67 ; I^{2} 44 \%$ ). When an outlier, Sabin et al.'s study [71], was omitted from overall pooled analysis (14 studies), the analysis became homogenous and the association remained positive albeit weaker (OR 1.35, 95\% CI 1.15 to $1.59 ; I^{2} 0 \%$ ). However, in subgroup analysis, the ten studies measuring adherence by pill count (MEMS) (omitting [71]) found a non-significant association with ART adherence (OR 1.17, 95\% CI 0.95-1.45) compared to the significant association among the five studies measuring adherence by self-report (OR 1.64, 95\% CI 1.28-2.11). When the three studies at low risk of bias were pooled in subgroup analysis [50, 63, 66], there was no association found between the text messaging arm and the standard care arm (OR 1.03, 95\% CI $\left.0.78-1.37 ; I^{2} 42 \%\right)$.

The five RCTs measuring adherence by self-report were homogenous. Heterogeneity in the ten studies measuring adherence by pill count $\left(I^{2} 56 \%\right)$ was moderate when all studies were combined. However, when the outlier study by Sabin et al. [71] (OR 7.45, 95\% CI 2.90 to 19.15) was omitted, the RCTs became homogenous $\left(I^{2} 0 \%\right)$, and the effect measure weakened to show no association (OR 1.17, 95\% CI 0.95 to 1.45 ).

\section{HIV treatment outcomes (Fig. 4)}

Uptake of CD4 testing One study by Dryden-Peterson et al. ( $n=366$; $11 \%$ attrition) [45] examined the effectiveness of text messaging on the uptake of CD4 cell count testing revealing no significant association (OR 1.41, 95\% CI 0.91 to 2.19).

Viral load suppression Five studies $(n=1607 ; 2 \%$ attrition) examined the effectiveness of SMS to support HIV viral suppression [52, 56, 63, 71, 72]. In pooled analysis, the five RCTs showed no association in virological suppression below the level of detection between intervention and control groups with low heterogeneity (OR $1.08,95 \%$ CI 0.81 to $1.44 ; I^{2} 22 \%$ ).
Presence of a new opportunistic infection One study [75] ( $n=200 ; 0 \%$ attrition) reported the presence of new opportunistic infections in HIV positive participants at 3 months post-intervention. This study showed that SMS was not associated with the incidence of new opportunistic infections (OR 1.56, 95\% CI 0.85 to 2.85 ).

CD4 cell count increase Two studies $(n=208 ; 15 \%$ attrition) $[56,71]$ examining the proportion of patients that had an increase in CD4 cell count when a text message was received found no association (OR 1.13, 95\% CI 0.62 to $2.06 ; I^{2} 0 \%$ ). In addition to these two studies, Maduka $(n=104)$ [54] measured pre- and post-intervention median CD4 cell counts among intervention and control groups and found a greater increase in CD4 cell counts in the text message group than the control group (SMS group increased from 193 cells/ml to 575.0 cells $/ \mathrm{ml}$ compared to the control group that saw an increase from 131.0 cells $/ \mathrm{ml}$ to 361.5 cells $/ \mathrm{ml}$; $p=0.007)$.

Uptake of CD4 testing One study $(n=366 ; 11 \%$ attrition) [45] examined the uptake of CD4 testing when SMS were sent to the intervention group compared to standard care found no association (OR 1.41. 95\% CI $0.91,2.19)$.

ART initiation before 30 weeks gestation One study ( $n=366 ; 11 \%$ attrition) [45] examining text messaging to support ART initiation before 30 weeks gestation showed no association between the intervention and standard care groups (OR 1.59, 95\% CI 0.98 to 2.60 ).

\section{Test of publication bias}

Medication adherence was the only outcome that had sufficient studies to conduct a test of publication bias. The funnel plot with the trim and fill method (Additional file 7) for the 15 medication adherence studies indicated asymmetry. The Egger test of small-study effects revealed an estimated bias coefficient of 1.53 (standard error $0.41 ; p=0.002$ ). The trim and fill method predicted that two RCTs were missing from this analysis.

\section{GRADE assessment}

We assessed all outcomes using the GRADE approach. Results for three of the most important outcomes are in Table 2. The remaining assessments are available in Additional file 8. The eight RCTs contributing data to the appointment adherence outcome showed serious risk of bias $[41,43,49,58,59,61,70]$. The majority of studies were single-blinded; however, the outcome assessment appeared free from bias because the investigators blinded outcome assessors. There was a lack of blinding of patients in studies, and only one study clearly used 


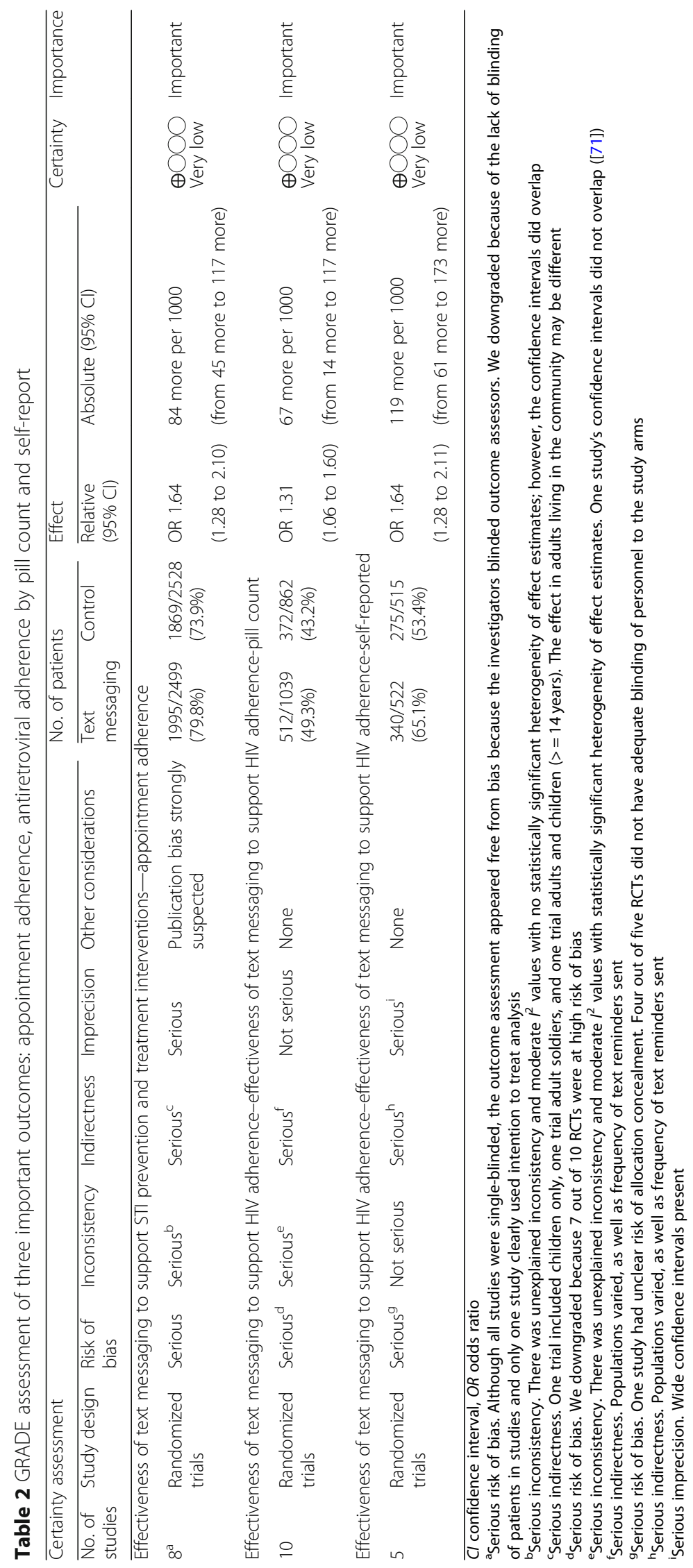


intention to treat analysis. Inconsistency and indirectness were assessed as serious because of moderate heterogeneity of effect estimates, and populations varied significantly. Imprecision was considered serious because of wide confidence intervals. Publication bias was strongly suspected. Appointment adherence was therefore assessed as very low-certainty evidence.

The ten studies contributing data to the adherence to ART by pill count outcome showed serious risk of bias $[42,47-50,56,63,66,71,72]$. Seven out of ten RCTs were assessed at high risk of bias due to unclear randomization and allocation concealment. There was unexplained inconsistency, with moderate heterogeneity, and one study's confidence intervals did not overlap [71]. Indirectness was assessed as serious because populations studied varied, as well as the frequency of text reminders sent. Adherence to ART by pill count was therefore assessed as very low-certainty evidence.

The five RCTs contributing data to the adherence to ART by self-report outcome showed serious risk of bias $[46,52,54,55,59]$ due to the unclear risk of allocation concealment (one study) and inadequate blinding of staff to the study arms (four out of five studies). Inconsistency was not serious as studies showed statistical homogeneity. Indirectness was serious because populations varied across studies, as did the frequency of text reminders sent. There was also serious imprecision because of wide confidence intervals. The adherence to ART by self-report outcome was therefore assessed as very low-certainty evidence.

\section{Deviations from planned protocol}

In our published protocol [31], we stated we would include randomized and nonrandomized controlled trials, pre- and post-test designs, observational (cross-sectional, case-series, case studies, and qualitative studies) that examined the effectiveness of SMS interventions on STIs. However, we only include RCTs in order to manage the large amount of data collected and because these represent the highest quality research. Similarly, we only reported on the 14 outcomes that were most dominant in the literature aimed at assessing the effectiveness of interventions (not qualitative outcomes such as acceptability). We also made our eligibility criteria broader than our originally planned criteria to allow for all types of comparisons (e.g., phone, emails) to be inclusive of other means of communicating health messages.

The initial search was conducted in 2013 in multiple databases (MEDLINE, ACP Journal Club, Database of Abstracts of Reviews, EMBASE, EBM Reviews, and Cochrane Library); however, the update search was conducted only in MEDLINE and mhealthevidence.org to contain the search results due to restricted resources available (staff and funding). We feel however that searching mhealthevidence.org in addition to MEDLINE would have retrieved the majority of randomized trials on this topic as it is a current database repository for all mHealth evidence implemented by Johns Hopkins Center for Communication Programs. Furthermore, in our more comprehensive search from 1996 to 2013 of five databases, we retrieved only 14 RCTs as compared to the additional 21 we retrieved from August 2013 to end of March 2017 (with consideration of the probable increase in SMS studies published) using the MEDLINE and mhealthevidence.org databases.

\section{Discussion}

\section{Summary of main results}

The purpose of this systematic review was to determine the effectiveness of SMS on STI/HIV prevention and control outcomes. Overall, the results of our systematic review highlight the equivocal nature of the evidence surrounding the effectiveness of SMS interventions of STI/HIV outcomes. SMS interventions vary greatly in different populations and when used for different purposes. SMS may be effective for some prevention interventions such as those aimed at improving appointment adherence (eight studies) and those aimed at increasing the uptake of circumcision (to prevent HIV) (two studies). They may also be effective in increasing adherence to HIV medications(15 studies) although this effect was primarily found in studies depending on self-report suggesting that social desirability bias may have come into play. Conversely, pooled results from seven studies indicate that there is no statistically significant association between providing SMS interventions and improving HIV treatment outcomes.

It should be noted that the certainty of the evidence was found to be low due to inconsistency, indirectness, and imprecision. This may be explained by the markedly variant in terms of SMS methods (one-way versus two-way, frequency of text messages sent, their content, and the populations targeted) among the studies we examined. This explanation is supported by others who have experienced disparate effects of SMS using identical study designs, geographic location, and SMS mode of delivery but among a slightly different population group [76]. Moreover, uncertainty among our results may be due to the high percentage of studies (75\%) having high risk of selection bias and performance bias due to inability to conceal randomization allocation and inability to blind study participants and study personnel.

\section{Significance of this review}

Our study points out that the evidence related to SMS interventions remains equivocal and individual studies should be interpreted with caution. Randomized controlled trials are considered the gold standard for 
determining the effectiveness of an intervention. However, when bias is introduced by lack of blinding, small sample sizes, high attrition rates, and unclear allocation concealment, confidence in the evidence is reduced. Our study highlights the challenges that are encountered when attempting to pool results from RCTs that are prone to bias. In addition, there is a need for standardized reporting, using common definitions for outcomes for RCTs aimed at evaluating SMS to reduce unexplained inconsistencies. Finally, we recommend authors of RCT studies to include statements about registration of study protocols in their published work to minimize concerns of reporting bias and include intention-to-treat analyses in their research to avoid bias resulting from crossover.

\section{Agreements and disagreements with other studies or reviews}

Our results are consistent with the findings of a 2013 Cochrane review $(n=8)$ that showed low to moderate certainty related to SMS improving attendance rates at any healthcare appointments [77]. Our findings also agree with a 2017 network meta-analysis [29] reporting results of a pairwise direct comparison of HIV treatment adherence using a SMS intervention versus standard care in four RCTs (OR of 1.70) (95\% CI 1.16, 2.49, certainty of the evidence, moderate). Another 2012 Cochrane review $(n=2)$ evaluated SMS for promoting adherence to antiretroviral therapy in patients with HIV conducted in Kenya only [27]. Our analysis included these two high-certainty studies and included an additional 13 RCTs.

Two other reviews pooled the results of RCTs and quasi-experimental controlled trials on the effectiveness of SMS interventions on HIV adherence and viral load $[26,28]$. One systematic review with meta-analysis by Mayer and Fontelo examined (1) appointment non-attendance and (2) HIV adherence when an SMS reminder was sent to participants in intervention studies compared to a control group or pre-intervention group [28]. They included both pre-post studies and randomized controlled trials but did not include a subgroup analysis by study design. The pooling of the Mayer and Fontelo data on non-attendance is somewhat counterintuitive as clinicians, researchers, and funders want to know if the addition of SMS reminders will increase appointment attendance at STI/HIV clinics. Further, Mayer and Fontelo's pooled analysis of the effectiveness of SMS reminders to improve medication adherence was done using a standardized mean difference. The majority of studies in our analysis $(67 \%, n=10 / 15)$ reported data as proportion of patients $90-95 \%$ adherent compared to control; therefore, we chose to analyze the HIV adherence data on a dichotomous scale. This decision, as well as the decision to pool only RCTs, resulted in a lower level of heterogeneity ( $I^{2} 44 \%$ over the 15 RCTs) in our pooled analysis compared to Mayer and Fontelo's [28] high heterogeneity $\left(I^{2} 99 \%\right)$. When performing sensitivity analyses, we omitted the one study that differed in its definition of adherence (patients were categorized as optimally and suboptimally adherent) which resulted in a homogenous analysis $\left(I^{2} 0 \%\right)$ and an OR of 1.35 with a narrow confidence interval (95\% CI 1.15-1.59).

Two other previous reviews are available. Lim et al. [78] provided a review of SMS interventions. However, this review is outdated (2008) and was limited to describing SMS programs. The authors acknowledge that the programs the report on were not rigorously evaluated. We are providing more current evidence that has been rigorously produced. Zou et al. [79] provided a review that is 4 years old and had a narrow scope. They evaluated the effect of SMS on screening rates for bacterial STIs among men who have sex with men. Our study provides a more comprehensive review of all STIs for all populations. The reference list for this review was utilized during our search strategy.

Systematic reviews by Chavez et al. [80], L'Engle et al. [24], and Zou et al. [81] present important findings with respect to text messaging and sexual health outcomes; however, they do not cover the effectiveness of SMS interventions for a broad spectrum of STI prevention and control strategies. Our review fills an important gap by including more RCTs. In addition, the scope of our review is wider because it provides evidence from developed and developing countries.

\section{Strengths and limitations of the present review}

A major strength of this present review is that we have included an evaluation of SMS for both STIs and HIV. This is important because public health clinicians do not separate out interventions based on these two things. Introduction of bias in our review was minimized by double independent screening and extraction, and assessing the risk of bias of each included study by two authors. Although no test of agreement was conducted between reviewers, all discrepancies were discussed and resolved.

The authors recognize that limiting our review to studies published in English may produce an unintended bias as SMS interventions conducted in non-Englishspeaking countries may have a different effect. Therefore, we caution readers from non-English speaking countries to take this into consideration when considering introducing SMS interventions in their respective jurisdictions. We did not include studies that could only be found in the grey literature (i.e., conference proceedings) as it is difficult to assess certainty and bias for these studies. This may have resulted in missing more 
recent studies which may have resulted in including studies with a greater effect size (due to possible increased comfort levels with text messaging). In addition, only one reviewer was involved in searching for studies and screening titles and abstracts for eligibility during phase 2. This may have resulted in some relevant studies spuriously being excluded. Moreover, GRADE assessments were conducted by a single reviewer, due to resource limitations, which may have biased the reporting in an unintended way. In addition, we did not report the results of one primary outcome-acceptability of SMS for STI interventions and one secondary outcomefeasibility of program delivery, which was reported by some RCTs in our sample. We felt that this information was better suited to a subsequent (second paper) reporting implementation results.

The initial search was conducted in 2013 in multiple databases (MEDLINE, ACP Journal Club, Database of Abstracts of Reviews, EMBASE, EBM Reviews, and Cochrane Library); however, the update search was conducted only in MEDLINE and mhealthevidence.org to contain the search results due to restricted resources available (staff and funding). We feel however that searching mhealthevidence.org in addition to MEDLINE would have retrieved the majority of randomized trials on this topic as it is a current database repository for all mHealth evidence implemented by Johns Hopkins Center for Communication Programs. Furthermore, in our more comprehensive search from 1996 to 2013 of five databases, we retrieved only 14 RCTs as compared to the additional 21 we retrieved from August 2013 to end of March 2017 (with consideration of the probable increase in SMS studies published) using the MEDLINE and mhealthevidence.org databases.

The studies we included were of varying certainty, and the certainty of the evidence on two important outcomes was considered low, thus limiting the confidence in the summary effect estimates. We were also only able to pool a sufficient number of high-certainty studies for one outcome-adherence to ART. Both certainty of evidence and study quality were considered when making conclusions, reducing the influence of lower certainty studies. In addition, rigorous searching of multiple databases was conducted; however, publication bias is suspected due to non-publication of many negative trials.

\section{Future research}

High certainty RCTs are required to adequately assess the effect of text messaging to support all STI/HIV prevention and treatment outcomes included in this review. Ongoing trials into text messaging support to STI/HIV interventions might provide more power to update of these meta-analyses [82, 83]. Research into messaging interventions should also include other messaging platforms including WhatsApp, Facebook, etc. and compare results with cell phone texting. Moreover, our study did not report reported adverse events related to the SMS interventions. This should be included in a future review. Future research should also examine whether reminders alone are as effective as reminders embedded in educative messages $[84,85]$. Finally, RCTs can be designed as double-blinded by delivering the same number of text messages but with different content to the patient and control groups, thus making the trials less prone to biased results.

\section{Application to clinical care}

SMS is a flexible method to deliver health messages as it allows for instantaneous delivery directly to individuals at any time, place, or setting via their phones [86]. However, it is important to evaluate the effectiveness of an intervention once it has been initiated. The use of digital technology in designing interventions aimed at improving health outcomes including those related to STI and HIV has been approached with great enthusiasm. However, as others have pointed out [80], digital technology is rapidly changing and therefore research that has been conducted with SMS interventions may soon only be useful for a short period of time.

\section{Conclusion}

The effectiveness of SMS interventions to improve STI/ HIV outcomes remains equivocal, and due to the lack of precision of our pooled results and inconsistency of findings due to patient characteristic variability, it is incumbent upon program planners to evaluate the effectiveness of any program to ensure it is achieving the intended result.

\section{Additional files}

\author{
Additional file 1: PRISMA Checklist. (DOCX $30 \mathrm{~kb}$ ) \\ Additional file 2: Ovid Search Strategy Phase 1. (DOCX 17 kb) \\ Additional file 3: Ovid Search Strategy Phase 2. (DOCX 18 kb) \\ Additional file 4: Eligibility Criteria. (DOCX 14 kb) \\ Additional file 5: Table of SMS Messages. (DOCX $21 \mathrm{~kb}$ ) \\ Additional file 6: Risk of Bias Figures. (DOCX $47 \mathrm{~kb}$ ) \\ Additional file 7: Publication Bias Analysis and Subgroup Analysis Table. \\ (DOCX $26 \mathrm{~kb}$ )
}

Additional file 8: GRADE Assessment of other Outcomes. (DOCX 18 kb)

\section{Abbreviations}

aOR: Adjusted odds ratio; ART: Antiretroviral therapy; Cl: Confidence interval; GRADE: Grading of Recommendations Assessment, Development and Evaluation; GUM: Genitourinary medicine; HIV: Human immunodeficiency virus; HPV: Human papillomavirus; HR: Hazard ratio; Interve.: Intervention; IRR: Incidence rate ratio; ITT: Intention-to-treat; MCO: Managed care organization; MEMS: Medication event monitoring system; MSM: Men who have sex with men; NR: Not reported; Ol: Opportunistic infections; OR: Odds ratio; PICO: Population, Intervention, Comparison, Outcome; PID: Pelvic inflammatory disease; 
RCTS: Randomized controlled trials; RR: Risk ratio; SD: Standard deviation; SMS: Short message service; SR: Systematic review; STI: Sexually transmitted infection; VAS: Visual analog scale

\section{Acknowledgements}

Not applicable

\section{Ethical approval and consent to participate}

Not applicable

\section{Funding}

This research was funded by the Canadian Institutes for Health Research (FRN 126321). CL was funded by an Australian Postgraduate Award and an International Postgraduate Research Scholarship administered through Monash University, Australia.

\section{Availability of data and materials}

All data generated or analyzed during this study are included in this published article.

\section{Authors' contributions}

Research question, study design, and protocol were formulated by $C L$, DT, $\mathrm{RL}, \mathrm{GO}, \mathrm{MG}$, and TW. Systematic review was conducted by $\mathrm{DT}, \mathrm{CL}, \mathrm{PL}$, OW, and JG. Writing/reviewing of the manuscript was done by all authors. All authors read and approved the final manuscript.

\section{Consent for publication}

Not applicable

\section{Competing interests}

Dr. Richard Lester (RL) has financial as well as professional interests in WelTel mHealth Society (non-profit organization) and WelTel Incorporated (private company). These two organizations develop the WelTel SMS technology platform. RL was an author of one of the included RCTs, but did not extract data and did not analyze the data. No other authors were authors of any of the included RCTs, and have no competing interests.

\section{Publisher's Note}

Springer Nature remains neutral with regard to jurisdictional claims in published maps and institutional affiliations.

\section{Author details}

'University of British Columbia, 1147 Research Road, Rm ARTS 154, Kelowna BC V1V 1V7, Canada. 'University of British Columbia, 2329 West Mall, Vancouver BC V6T 1Z4, Canada. ${ }^{3}$ BC Centre for Disease Control, 655 W12th Ave, Vancouver, BC, Canada. ${ }^{4}$ Vancouver Island Health Authority, 1952 Bay Street, Victoria, BC V8R 1J8, Canada. ${ }^{5}$ Health Canada/Santé Canada, 200 Eglantine Driveway, Room 1913A, Ottawa, ON K1A OK9, Canada. ${ }^{6}$ University of British Columbia, Research Pavilion, Rm 566, 828 W 10th, Vancouver, BC V5Z 1 M9, Canada. 'University of British Columbia, Box 42, 4500 Oak Street, Vancouver, BC V6H 3N1, Canada.

\section{Received: 16 September 2018 Accepted: 18 December 2018}

Published online: 08 January 2019

\section{References}

1. World Health Organization. Sexually transmitted infections (STIs): Key facts. 2016 [cited 2018 Jul 17]. Available from: http://www.who.int/news-room/ fact-sheets/detail/sexually-transmitted-infections-(stis).

2. BC Centre for Disease Control. STI annual report. 2015. Vancouver: BC Centre for Disease Control; [cited 2018 Jul 15]. Available from: http://www. bccdc.ca/resource-gallery/Documents/Statistics\%20and\%20Research/ Statistics\%20and\%20Reports/STI/STI_Annual_Report_2015-FINAL.pdf

3. Kirby Institute. HIV viral hepatitis and sexually transmissible infections in Australia: annual surveillance report. Sydney: Kirby Institute, UNSW; 2017. Available from: https://kirby.unsw.edu.au/sites/default/files/kirby/report/ SERP_Annual-Surveillance-Report-2017_compressed.pdf

4. Abara WE, Hess KL, Neblett Fanfair R, Bernstein KT, Paz-Bailey G. Syphilis trends among men who have sex with men in the United States and Western Europe: a systematic review of trend studies published between 2004 and 2015. PLoS One. 2016;11(7):e0159309.
5. Executive Summary. Report on sexually transmitted infections in Canada: 2009. Ottawa: Government of Canada; 2009 [cited 2018 Jul 17]. Available from: https://www.canada.ca/en/public-health/services/ infectious-diseases/surveillance-epidemiology-sexually-transmittedinfections-hep-b-c/executive-summary-report-2009.html

6. Savage EJ, Hughes $G$, Ison C, Lowndes CM, European surveillance of sexually transmitted infections network. Syphilis and gonorrhoea in men who have sex with men: a European overview. Euro Surveill Bull Eur Sur Mal Transm Eur Commun Dis Bull. 2009;26, 14(47).

7. Siegel J. Appendix D: Estimates of the economic burden of STDs: Review of the literature with updates. In: Eng T, Butler W, editors. The HIdden Epidemic: confronting sexually transmitted diseases. Wahington (DC): National Academics Press (US); 1997.

8. Iribarren SJ, Cato K, Falzon L, Stone PW. What is the economic evidence for mHealth? A systematic review of economic evaluations of mHealth solutions. PLoS One. 2017;12(2):e0170581.

9. Patel AR, Kessler J, Braithwaite RS, Nucifora KA, Thirumurthy $H$, Zhou $\mathrm{O}$ et al. Economic evaluation of mobile phone text message interventions to improve adherence to HIV therapy in Kenya. Medicine (Baltimore). 2017;96(7):e6078

10. Steen R, Elvira Wi T, Kamali A, Ndowa F. Control of sexually transmitted infections and prevention of HIV transmission: mending a fractured paradigm. Bull World Health Organ. 2009;87(11):858-65.

11. Statista: The Statistics Portal. Number of mobile phone users worldwide from 2015 to 2020 (in billions). 2018 [cited 2018 Jul 17]. Available from: https://www. statista.com/statistics/274774/forecast-of-mobile-phone-users-worldwide/

12. We Are Social and Hootsuite: Digital in 2017 Global Overview Report. New York; 2017. Available from: https://wearesocial.com/special-reports/digital-in2017-global-overview. Accessed 24 Dec 2018.

13. van der Kop ML, Memetovic J, Patel A, Marra F, Sadatsafavi M, Hajek J, et al. The effect of weekly text-message communication on treatment completion among patients with latent tuberculosis infection: study protocol for a randomised controlled trial (WelTel LTBI). BMJ Open. 2014; 4(4):e004362.

14. Harris LT, Lehavot K, Huh D, Yard S, Andrasik MP, Dunbar PJ, et al. Two-way text messaging for health behavior change among human immunodeficiency virus-positive individuals. Telemed J E-Health Off J Am Telemed Assoc. 2010;16(10):1024-9.

15. Lewis MA, Uhrig JD, Bann CM, Harris JL, Furberg RD, Coomes C, et al. Tailored text messaging intervention for HIV adherence: a proof-of-concept study. Health Psychol Off J Div Health Psychol Am Psychol Assoc. 2013; 32(3):248-53.

16. Wald DS, Butt S, Bestwick JP. One-way versus two-way text messaging on improving medication adherence: meta-analysis of randomized trials. Am J Med. 2015:128(10):1139.e1-5.

17. Blackburn L, Blatnik A. Promoting sexual health with SMS texting technology. Nurs Womens Health. 2013:17(6):465-9.

18. Lim MSC, Sacks-Davis R, Aitken CK, Hocking JS, Hellard ME. Randomised controlled trial of paper, online and SMS diaries for collecting sexual behaviour information from young people. J Epidemiol Community Health. 2010;64(10):885-9.

19. Gold J, Lim MSC, Hellard ME, Hocking JS, Keogh L. What's in a message? Delivering sexual health promotion to young people in Australia via text messaging. BMC Public Health. 201029;10:792.

20. Perry RCW, Kayekjian KC, Braun RA, Cantu M, Sheoran B, Chung PJ. Adolescents' perspectives on the use of a text messaging service for preventive sexual health promotion. J Adolesc Health Off Publ Soc Adolesc Med. 2012:51(3):220-5.

21. Robotham D, Satkunanathan S, Reynolds J, Stahl D, Wykes T. Using digital notifications to improve attendance in clinic: systematic review and metaanalysis. BMJ Open. 2016;6(10):e012116.

22. Cornelius JB, Appiah JA. Using mobile technology to promote safe sex and sexual health in adolescents: current practices and future recommendations. Adolesc Health Med Ther. 2016:7:43-57.

23. Jones $K$, Eathington $P$, Baldwin K, Sipsma $H$. The impact of health education transmitted via social media or text messaging on adolescent and young adult risky sexual behavior: a systematic review of the literature. Sex Transm Dis. 2014:41(7):413-9.

24. L'Engle KL, Mangone ER, Parcesepe AM, Agarwal S, Ippoliti NB. Mobile phone interventions for adolescent sexual and reproductive health: a systematic review. Pediatrics. 2016;138(3). 
25. Desai M, Woodhall SC, Nardone A, Burns F, Mercey D, Gilson R. Active recall to increase HIV and STI testing: a systematic review. Sex Transm Infect. 2015; 91(5):314-23.

26. Finitsis DJ, Pellowski JA, Johnson BT. Text message intervention designs to promote adherence to antiretroviral therapy (ART): a meta-analysis of randomized controlled trials. PLoS One. 2014;9(2):e88166.

27. Horvath T, Azman H, Kennedy GE, Rutherford GW. Mobile phone text messaging for promoting adherence to antiretroviral therapy in patients with HIV infection. Cochrane Database Syst Rev. 2012;3:CD009756.

28. Mayer JE, Fontelo P. Meta-analysis on the effect of text message reminders for HIV-related compliance. AIDS Care. 2017;29(4):409-17.

29. Kanters S, Park JJH, Chan K, Socias ME, Ford N, Forrest Jl, et al. Interventions to improve adherence to antiretroviral therapy: a systematic review and network meta-analysis. Lancet HIV. 2017;4(1):e31-40.

30. Guyatt GH, Oxman AD, Vist GE, Kunz R, Falck-Ytter Y, Alonso-Coello P, et al. GRADE: an emerging consensus on rating quality of evidence and strength of recommendations. BMJ. 2008:336(7650):924-6.

31. Lunny C, Taylor D, Memetovic J, Wärje O, Lester R, Wong T, et al. Short message service (SMS) interventions for the prevention and treatment of sexually transmitted infections: a systematic review protocol. Syst Rev. 2014;3:7.

32. Moher D, Liberati A, Tetzlaff J, Altman DG, PRISMA Group. Preferred reporting items for systematic reviews and meta-analyses: the PRISMA statement. J Clin Epidemiol. 2009;62(10):1006-12.

33. Higgins J, Altman D, editors. Chapter 8: Assessing risk of bias in included studies. In: Cochran Handbook for Systematic Reviews of Interventions: 5.2. The Cochrane Collaboration; 2018. Available from: http:/handbook-5-1.cochrane.org/.

34. McMaster University. GRADEpro G: GRADEpro Guideline Development Tool. 2015.

35. StataCorp. Stata Statistical Software: Version 13. College STatopm: StataCorp LP; 2011.

36. Harris R, Bradburn M, Deeks J. Metan: fixed-and random-effects metaanalysis. Stata J. 2008;8(8).

37. Sterne JA, Harbord RM. Funnel plots in meta-analysis. Stata J. 2004;4:127-41.

38. Harbord RM, Harris RJ, Sterne JA. Updated tests for small-study effects in meta-analyses. Stata J. 2009;9:197-210.

39. Steichen TJ. Nonparametric trim and fill analysis of publication bias in metaanalysis: erratum. Stat Tech Bull. 2001;10 Available from: https://econpapers. repec.org/article/tsjstbull/.

40. Barnabas RV, van Rooyen H, Tumwesigye E, Brantley J, Baeten JM, van Heerden A, et al. Uptake of antiretroviral therapy and male circumcision after community-based HIV testing and strategies for linkage to care versus standard clinic referral: a multisite, open-label, randomised controlled trial in South Africa and Uganda. Lancet HIV. 2016;3(5):e212-20.

41. Bigna JJR, Noubiap JJN, Kouanfack C, Plottel CS, Koulla-Shiro S. Effect of mobile phone reminders on follow-up medical care of children exposed to or infected with HIV in Cameroon (MORE CARE): a multicentre, single-blind, factorial, randomised controlled trial. Lancet Infect Dis. 2014 Jul;14(7):600-8.

42. da Costa TM, Barbosa BJP, Gomes e Costa DA, Sigulem D, de Fátima Marin $H$, Filho AC, et al. Results of a randomized controlled trial to assess the effects of a mobile SMS-based intervention on treatment adherence in HIV/ AIDS-infected Brazilian women and impressions and satisfaction with respect to incoming messages. Int J Med Inf. 2012;81(4):257-69.

43. Davey J, Nhavoto JA, Augusto O, Ponce W, Traca D, Nguimfack A, et al. SMSaúde: evaluating mobile phone text reminders to improve retention in HIV care for patients on antiretroviral therapy in Mozambique. J Acquir Immune Defic Syndr 1999. 2016;73(2):e23-30.

44. de Tolly K, Skinner D, Nembaware V, Benjamin P. Investigation into the use of short message services to expand uptake of human immunodeficiency virus testing, and whether content and dosage have impact. Telemed J EHealth Off J Am Telemed Assoc. 2012;18(1):18-23.

45. Dryden-Peterson S, Bennett K, Hughes MD, Veres A, John O, Pradhananga R, et al. An augmented SMS intervention to improve access to antenatal CD4 testing and ART initiation in HIV-infected pregnant women: a cluster randomized trial. PLoS One. 2015;10(2):e0117181

46. Garofalo R, Kuhns LM, Hotton A, Johnson A, Muldoon A, Rice D. A randomized controlled trial of personalized text message reminders to promote medication adherence among HIV-positive adolescents and young adults. AIDS Behav. 2016;20(5):1049-59.

47. Haberer JE, Musiimenta A, Atukunda EC, Musinguzi N, Wyatt MA, Ware NC, et al. Short message service (SMS) reminders and real-time adherence monitoring improve antiretroviral therapy adherence in rural Uganda. AIDS Lond Engl. 2016;30(8):1295-300.

48. Hardy H, Kumar V, Doros G, Farmer E, Drainoni M-L, Rybin D, et al. Randomized controlled trial of a personalized cellular phone reminder system to enhance adherence to antiretroviral therapy. AIDS Patient Care STDs. 2011;25(3):153-61.

49. Ingersoll KS, Dillingham RA, Hettema JE, Conaway M, Freeman J, Reynolds $\mathrm{G}$, et al. Pilot RCT of bidirectional text messaging for ART adherence among nonurban substance users with HIV. Health Psychol Off J Div Health Psychol Am Psychol Assoc. 2015;34S:1305-15.

50. Kalichman SC, Kalichman MO, Cherry C, Swetzes C, Amaral CM, White D, et al. Brief behavioral self-regulation counseling for HIV treatment adherence delivered by cell phone: an initial test of concept trial. AIDS Patient Care STDs. 2011;25(5):303-10.

51. Kempe A, O'Leary ST, Shoup JA, Stokley S, Lockhart S, Furniss A, et al. Parental choice of recall method for HPV vaccination: a pragmatic trial. Pediatrics. 2016;137(3):e20152857.

52. Lester RT, Ritvo P, Mills EJ, Kariri A, Karanja S, Chung MH, et al. Effects of a mobile phone short message service on antiretroviral treatment adherence in Kenya (WelTel Kenya1): a randomised trial. Lancet Lond Engl. 2010; 376(9755):1838-45.

53. Lim MSC, Hocking JS, Aitken CK, Fairley CK, Jordan L, Lewis JA, et al. Impact of text and email messaging on the sexual health of young people: a randomised controlled trial. J Epidemiol Community Health. 2012;66(1):69-74

54. Maduka O, Tobin-West $\mathrm{Cl}$. Adherence counseling and reminder text messages improve uptake of antiretroviral therapy in a tertiary hospital in Nigeria. Niger J Clin Pract. 2013;16(3):302-8.

55. Mbuagbaw L, Thabane L, Ongolo-Zogo P, Lester RT, Mills EJ, Smieja M, et al. The Cameroon Mobile Phone SMS (CAMPS) trial: a randomized trial of text messaging versus usual care for adherence to antiretroviral therapy. PLoS One. 2012;7(12):e46909.

56. Moore DJ, Poquette A, Casaletto KB, Gouaux B, Montoya JL, Posada C, et al. Individualized texting for adherence building (iTAB): improving antiretroviral dose timing among HIV-infected persons with co-occurring bipolar disorder. AIDS Behav. 2015;19(3):459-71.

57. Morris J, Wang W, Wang L, Peddecord KM, Sawyer MH. Comparison of reminder methods in selected adolescents with records in an immunization registry. J Adolesc Health Off Publ Soc Adolesc Med. 2015:56(5 Suppl):S27-32.

58. Mugo PM, Wahome EW, Gichuru EN, Mwashigadi GM, Thiong'o AN, Prins $H A B$, et al. Effect of text message, phone call, and in-person appointment reminders on uptake of repeat HIV testing among outpatients screened for acute HIV infection in Kenya: a randomized controlled trial. PLoS One. 2016;11(4):e0153612.

59. Norton BL, Person AK, Castillo C, Pastrana C, Subramanian M, Stout JE. Barriers to using text message appointment reminders in an HIV clinic. Telemed J E-Health Off J Am Telemed Assoc. 2014;20(1):86-9.

60. Nsagha DS, Lange I, Fon PN, Nguedia Assob JC, Tanue EA. A randomized controlled trial on the usefulness of mobile text phone messages to improve the quality of care of HIV and AIDS patients in Cameroon. Open AIDS J. 2016;10:93-103.

61. Odeny TA, Bailey RC, Bukusi EA, Simoni JM, Tapia KA, Yuhas K, et al. Text messaging to improve attendance at post-operative clinic visits after adult male circumcision for HIV prevention: a randomized controlled trial. PLoS One. 2012;7(9):e43832

62. Odeny TA, Bukusi EA, Cohen CR, Yuhas K, Camlin CS, McClelland RS. Texting improves testing: a randomized trial of two-way SMS to increase postpartum prevention of mother-to-child transmission retention and infant HIV testing. AIDS Lond Engl. 2014;28(15):2307-12.

63. Orrell C, Cohen K, Mauff K, Bangsberg DR, Maartens G, Wood R. A randomized controlled trial of real-time electronic adherence monitoring with text message dosing reminders in people starting first-line antiretroviral therapy. J Acquir Immune Defic Syndr 1999. 2015;70(5):495-502.

64. Patel A, Stern L, Unger Z, Debevec E, Roston A, Hanover R, et al. Staying on track: a cluster randomized controlled trial of automated reminders aimed at increasing human papillomavirus vaccine completion. Vaccine. 2014;32(21):2428-33.

65. Perron NJ, Dao MD, Kossovsky MP, Miserez V, Chuard C, Calmy A, et al. Reduction of missed appointments at an urban primary care clinic: a randomised controlled study. BMC Fam Pract. 2010;11:79. 
66. Pop-Eleches C, Thirumurthy H, Habyarimana JP, Zivin JG, Goldstein MP de Walque $\mathrm{D}$, et al. Mobile phone technologies improve adherence to antiretroviral treatment in a resource-limited setting: a randomized controlled trial of text message reminders. AIDS Lond Engl. 2011;25(6):825-34

67. Rand CM, Brill H, Albertin C, Humiston SG, Schaffer S, Shone LP, et al. Effectiveness of centralized text message reminders on human papillomavirus immunization coverage for publicly insured adolescents. J Adolesc Health Off Publ Soc Adolesc Med. 2015;56(5 Suppl):S17-20.

68. Rand CM, Vincelli P, Goldstein NPN, Blumkin A, Szilagyi PG. Effects of phone and text message reminders on completion of the human papillomavirus vaccine series. J Adolesc Health Off Publ Soc Adolesc Med. 2017;60(1):113-9.

69. Richman AR, Maddy L, Torres E, Goldberg EJ. A randomized intervention study to evaluate whether electronic messaging can increase human papillomavirus vaccine completion and knowledge among college students. J Am Coll Health J ACH. 2016;64(4):269-78.

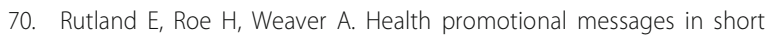
message service (SMS) follow-up of GU medicine clinic defaulters; a tool to improve subsequent attendance rates? Sex Transm Infect. 2012;88:A4-5.

71. Sabin LL, Bachman DeSilva M, Gill CJ, Zhong L, Vian T, Xie W, et al. Improving adherence to antiretroviral therapy with triggered real-time text message reminders: the China adherence through technology study. J Acquir Immune Defic Syndr 1999. 2015;69(5):551-9.

72. Shet A, De Costa A, Kumarasamy N, Rodrigues R, Rewari BB, Ashorn P, et al. Effect of mobile telephone reminders on treatment outcome in HIV: evidence from a randomised controlled trial in India. BMJ. 2014;349:95978.

73. Suffoletto B, Akers A, McGinnis KA, Calabria J, Wiesenfeld HC, Clark DB. A sex risk reduction text-message program for young adult females discharged from the emergency department. J Adolesc Health Off Publ Soc Adolesc Med. 2013;53(3):387-93.

74. Downing SG, Cashman C, McNamee H, Penney D, Russell DB, Hellard ME. Increasing chlamydia test of re-infection rates using SMS reminders and incentives. Sex Transm Infect. 2013;89(1):16-9.

75. Mbuagbaw L, Mursleen S, Lytvyn L, Smieja M, Dolovich L, Thabane L. Mobile phone text messaging interventions for HIV and other chronic diseases: an overview of systematic reviews and framework for evidence transfer. BMC Health Serv Res. 2015;15:33.

76. Fox MP, Kaufman JS. The WelTel Trial in context and the importance of null findings. Lancet Public Health. 2018;3(3):e107-8.

77. Gurol-Urganci I, de Jongh T, Vodopivec-Jamsek V, Atun R, Car J. Mobile phone messaging reminders for attendance at healthcare appointments. Cochrane Database Syst Rev. 2013;12:CD007458.

78. Lim MSC, Hocking JS, Hellard ME, Aitken CK. SMS STI: a review of the uses of mobile phone text messaging in sexual health. Int J STD AIDS 2008;19(5):287-90

79. Zou H, Fairley CK, Guy R, Chen MY. The efficacy of clinic-based interventions aimed at increasing screening for bacterial sexually transmitted infections among men who have sex with men: a systematic review. Sex Transm Dis. 2012;39(5):382-7.

80. Chávez NR, Shearer LS, Rosenthal SL. Use of digital media technology for primary prevention of STIS/HIV in youth. J Pediatr Adolesc Gynecol. 2014;27(5):244-57

81. Zou H, Fairley CK, Guy R, Bilardi J, Bradshaw CS, Garland SM, et al. Automated, computer generated reminders and increased detection of gonorrhoea, chlamydia and syphilis in men who have sex with men. PLoS One. 2013;8(4):e61972.

82. Myer, L. Identifier NCT01933477: Strategies to Optimize Antiretrovial Therapy Services for Maternal \& Child Health: the MCH-ART Study (MCH-ART). ClinicalTrials.gov. Available from: https://clinicaltrials.gov/ct2/show/ NCT01933477 [cited 24 Dec 2018]. 6 July 2016.

83. Pactr.org I: Identifier PACTR201507001178614: Prescott, M., Boeke, C. Gotora, T., William, H., Wadzanai, M., Justin, M., Alexio, G., McCarthy, M.E. Integration of EPI and pediatric HIV services for improved ART initiation. Pan African Clinical Trials Registry; South African Cochrane Centre. 2017.

84. Belzer ME, Kolmodin MacDonell K, Clark LF, Huang J, Olson J, Kahana SY, et al. Acceptability and feasibility of a cell phone support intervention for youth living with HIV with nonadherence to antiretroviral therapy. AIDS Patient Care STDs. 2015;29(6):338-45.
85. Belzer ME, Naar-King S, Olson J, Sarr M, Thornton S, Kahana SY, et al. The use of cell phone support for non-adherent HIV-infected youth and young adults: an initial randomized and controlled intervention trial. AIDS Behav. 2014;18(4):686-96.

86. Binagwaho A, Mugwaneza P, Irakoze AA, Nsanzimana S, Agbonyitor M, Nutt CT, et al. Scaling up early infant diagnosis of HIV in Rwanda, 2008-2010. J Public Health Policy. 2013;34(1):2-16.

\section{Ready to submit your research? Choose BMC and benefit from:}

- fast, convenient online submission

- thorough peer review by experienced researchers in your field

- rapid publication on acceptance

- support for research data, including large and complex data types

- gold Open Access which fosters wider collaboration and increased citations

- maximum visibility for your research: over $100 \mathrm{M}$ website views per year

At BMC, research is always in progress.

Learn more biomedcentral.com/submissions 